\title{
Recent Hubble Space Telescope Observations of SN 1987A: Broad Emission Lines
}

\author{
Kevin France \\ Center for Astrophysics and Space Astronomy, University of Colorado, 389 UCB, Boulder, \\ CO 80309; kevin.france@colorado.edu
}

\begin{abstract}
Observations with the Hubble Space Telescope (HST), conducted since 1990, have allowed us to create a "movie" of the evolution of the core-collapse supernova SN 1987A from 3-25 years after the explosion. Critical to understanding the late time evolution of SN 1987A was the successful HST Servicing Mission 4 in May 2009. The repair of the STIS instrument and the installation of the WFC3 imager and COS spectrograph have provided crucial data points for understanding the temporal variability in the physical structure and energy sources for SN $1987 \mathrm{~A}$, as well as measurements of the chemical abundances of the ejecta. In this proceeding, I will focus on two topics that have made use of the expanded capability of HST and highlight the importance of access to a UV/optical space observatory for the studies of local supernovae: 1) 2) The decreasing maximum velocity of neutral hydrogen crossing the reverse shock front and the role of soft X-ray/EUV heating in the outer supernova debris and 2) The detection of metals $\left(\mathrm{N}^{4+}\right.$ and $\mathrm{C}^{3+}$ ions) crossing the reverse shock front and CNO processing in the progenitor star.
\end{abstract}

Keywords. circumstellar matter, shock waves, supernovae: individual: SN 1987A

\section{Introduction}

Borkowski, Blondin, \& McCray (1997) predicted that the spectrum of SN 1987A should display very broad ( $\Delta v \sim \pm 12,000 \mathrm{~km} \mathrm{~s}^{-1}$ ) emission lines of Ly $\alpha, \mathrm{H} \alpha, \mathrm{NV} \lambda 1240$, and HeII $\lambda 1640$, produced where the freely expanding supernova debris crosses a reverse shock located inside the equatorial circumstellar ring. In September 1997, using the Hubble Space Telescope-Space Telescope Imaging Spectrograph (HST-STIS), Sonneborn et al. (1998) detected broad Ly $\alpha$ emission, and Michael et al. (1998) showed how observations of this emission can be used to map the shape of the reverse shock and the flux of HI atoms crossing it. Michael et al. (2003) and Heng et al. (2006) analyzed subsequent (February 1999 - October 2002) STIS observations of both Ly $\alpha$ and $\mathrm{H} \alpha$ to map the increasing flux of HI atoms across the reverse shock.

In this proceeding, I will summarize recent analyses of the broad emission features coming from the outer debris and the reverse shock region of SN 1987A. We refer readers to France et al. (2010) and France et al. (2011) for additional details related to the observations, spectral reduction, and data analysis of the HST observations of SN 1987A.

\section{Observations}

SN 1987A was observed with the medium resolution far-UV modes of HST-COS (G130M and G160M) on 2011 February 11 and March 14 for a total of 7 spacecraft orbits (18555 s; Table 1) as part of the Supernova 1987A INTensive Study (SAINTS GO12241; PI - R. Kirshner). A description of the COS instrument and on-orbit performance characteristics can be found in Osterman et al. (2011). Star 3 contributes negligible flux to the one-dimensional spectrum. The custom data extractions were then reprocessed with the COS calibration pipeline, CALCOS v2.13.6, and combined with the custom IDL 


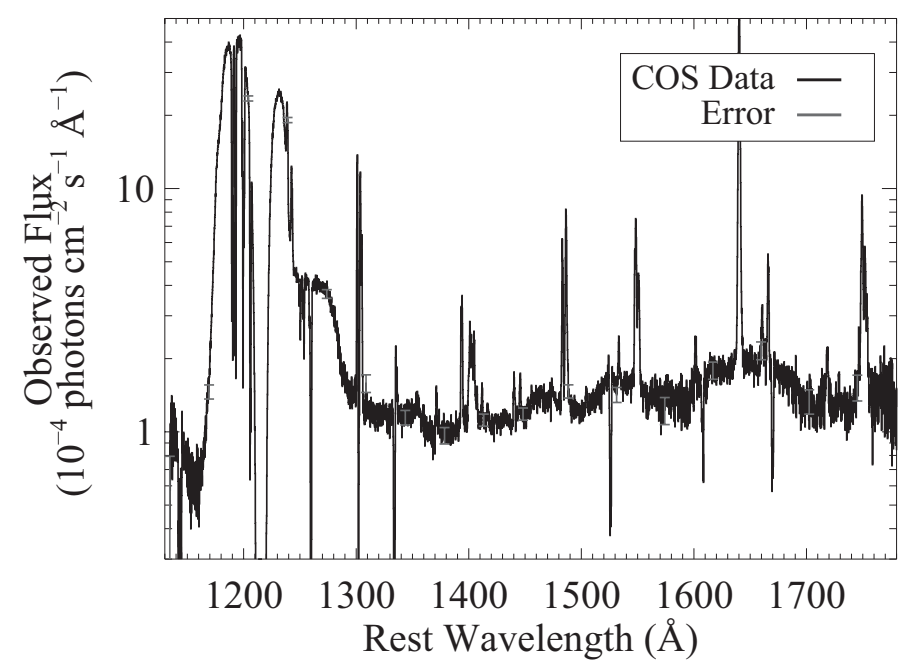

Figure 1. Full far-UV spectrum of SN 1987A, obtained with the Cosmic Origins Spectrograph, aboard Hubble. Broad emission from the SN 1987A reverse shock, narrow emission lines attributable to circumstellar hotspots, as well as underlying continuum emission are observed. Representative 1- $\sigma$ error bars (a combination of photon statistics and flux calibration uncertainties) are shown in red.

coaddition procedure described by Danforth et al. (2010). Data were obtained in four central wavelength settings in each far-UV grating mode $(\lambda 1291,1300,1309$, and 1318 with G130M and $\lambda 1577,1589,1600$, and 1611 with G160M) at the default focal-plane split position. Observations at multiple wavelength settings provide continuous spectral coverage over the 1136 - $1782 \AA$ bandpass and minimize the residual fixed pattern noise from the detector grid wires and the MCP pores. The point source resolving power of the medium resolution COS far-UV modes is $R \equiv \Delta \lambda / \lambda \approx 18,000\left(\Delta v=17 \mathrm{~km} \mathrm{~s}^{-1}\right)$; however, the filled-aperture resolving power is $R \sim 1500\left(\Delta v \sim 200 \mathrm{~km} \mathrm{~s}^{-1}\right.$; France et al. 2009).

The STIS observations were obtained in the G140L mode, through the $52^{\prime \prime} \times 0.2^{\prime \prime}$ slit, on 31 January 2010. These data were the first ultraviolet observations of SN 1987A following the Hubble Servicing Mission 4. Approximately three HST orbits worth of data were acquired at a resolving power $R \sim 1500$, at $\sim 0.1^{\prime \prime}$ angular resolution across the 1150 - $1710 \AA$ bandpass. STIS G750L observations in the red were also obtained with somewhat lower spectral resolution, although with a factor of $\sim 2$ higher angular resolution.

\section{3. $\operatorname{Ly} \alpha, 2$ - photon Continuum, and Heating of the Outer Ejecta}

Figures 1, 2, and 4 show the broad emission from H I; Ly $\alpha$ and $\mathrm{H} \alpha$ line emission. The velocity distribution of the neutral hydrogen emission in 2011 (Figure 1) extends from $-13000-+8000 \mathrm{~km} \mathrm{~s}^{-1}$. The velocity maxima are much smaller than the initial observations by Sonneborn et al. $\left(1998 ; \pm 20000 \mathrm{~km} \mathrm{~s}^{-1}\right)$ and are consistent with the decrease in maximum projected velocity observed from 2004 to 2010 (France et al. 2010). Previous work noted that the $\mathrm{Ly} \alpha / \mathrm{H} \alpha$ ratios from the reverse shock exceed the 5:1 photon production ratio expected for a Balmer-dominated shock (Heng et al. 2006; Heng $\&$ McCray 2007). France et al. (2010) found $\mathrm{Ly} \alpha / \mathrm{H} \alpha$ ratios $\geqslant 30$ from $-8000--2500$ $\mathrm{km} \mathrm{s}^{-1}$ and $\geqslant 20$ from $+3000-+7000 \mathrm{~km} \mathrm{~s}^{-1}$ for isolated cuts across the northern 


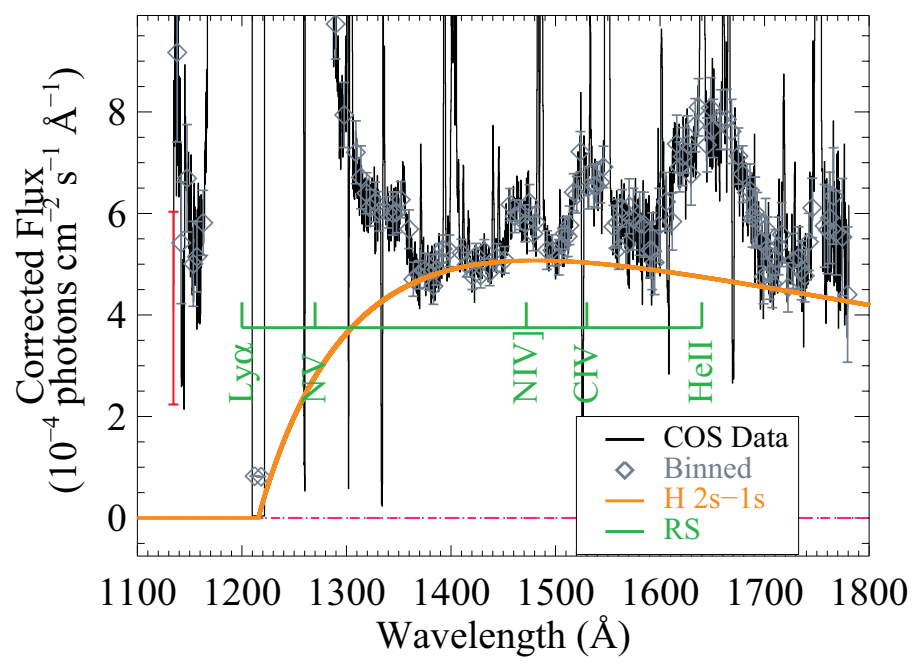

Figure 2. The extracted, one-dimensional spectrum of SN 1987A (black) and the spectrum averaged over $0.8 \AA$ bins chosen to avoid narrow features from hotspot emission and interstellar absorption. These data have been corrected for interstellar neutral hydrogen absorption $\left(\log _{10}(\mathrm{~N}(\mathrm{H}))=21.43\right)$ and interstellar dust extinction, assuming $E(B-V)=0.19$ and $R_{V}=3.1$. The broad spectral features are produced by a combination of hydrogen 2-photon emission $(2 s \rightarrow 1 s)$ and ionic emission from the reverse shock. A theoretical hydrogen 2-photon spectrum is shown overplotted in orange. Reverse shock emission features are identified in green.

and southern sides of the reverse shock, respectively. They attributed the enhanced Ly $\alpha$ emission to a second source of Ly $\alpha$ photons. They argued that Ly $\alpha$ photons from the hotspots are resonantly scattered by onrushing hydrogen with a distribution of velocities spanning a width $\Delta v_{H} \sim 3000-9000 \mathrm{~km} \mathrm{~s}^{-1}$ (unlike Ly $\alpha, \mathrm{H} \alpha$ is not a resonance line and therefore hotspot $\mathrm{H} \alpha$ photons pass through the debris freely).

While the Ly $\alpha$ enhancement at the largest negative velocities can likely be explained by this mechanism, a more likely physical scenario is that the lower velocity $( \pm 7000$ $\mathrm{km} \mathrm{s}^{-1}$ ) Ly $\alpha$ excess is attributable to the X-ray heating of the outer supernova debris. The low-density gas in the outer debris $\left(n_{H} \sim 100 \mathrm{~cm}^{3}\right.$; Smith et al. 2005; Heng et al. 2006) is heated by X-rays emitted from the shocked gas near the hotspots. In particular, the energy deposition of the soft X-ray/EUV photons from the shocked hotspots will be concentrated in a layer near the reverse shock (Fransson et al. 2011). When the ionization fraction is $\gtrsim 3 \times 10^{-2}$, the majority of the X-ray energy heats the gas through Coulomb stopping of fast photoelectrons (Xu et al. 1992; Kozma \& Fransson 1992). The primary coolants for this gas are $\operatorname{Ly} \alpha$ and 2-photon emission.

In this scenario, we expect to detect H I 2-photon emission from the same regions of enhanced Ly $\alpha$ production. The high sensitivity of the COS observations reveal this emission for the first time (Figure 2). The total integrated $(1216 \AA-6 \mu \mathrm{m}) \mathrm{H} \mathrm{I} 2$ photon flux is $9.1 \pm 0.6 \times 10^{-1}$ photons $\mathrm{cm}^{-2} \mathrm{~s}^{-1}$, and the observed ratio of Ly $\alpha$ line emission to 2-photon is $F(\operatorname{Ly} \alpha) / F(2 s)=1.96 \pm 0.23$. Since this ratio is consistent with the $2 p / 2 s$ ratio of 2.1 (observed as $F(\operatorname{Ly} \alpha) / F(2 s)$ ) expected for recombination at $T \approx 10^{4}$ $\mathrm{K}$ (Spitzer 1978) and the $(1 s \rightarrow 2 p) /(1 s \rightarrow 2 s)$ ratio of $2.05-2.10$ expected for excitation by thermal electrons (Callaway 1988), we infer that it is likely that the Ly $\alpha$ and the 2photon emission come from the same source. At the densities of the recombination regions in the hotspots $\left(n_{H}>4 \times 10^{6} \mathrm{~cm}^{-3}\right.$; Groningsson et al. 2008), the 2-photon emission 


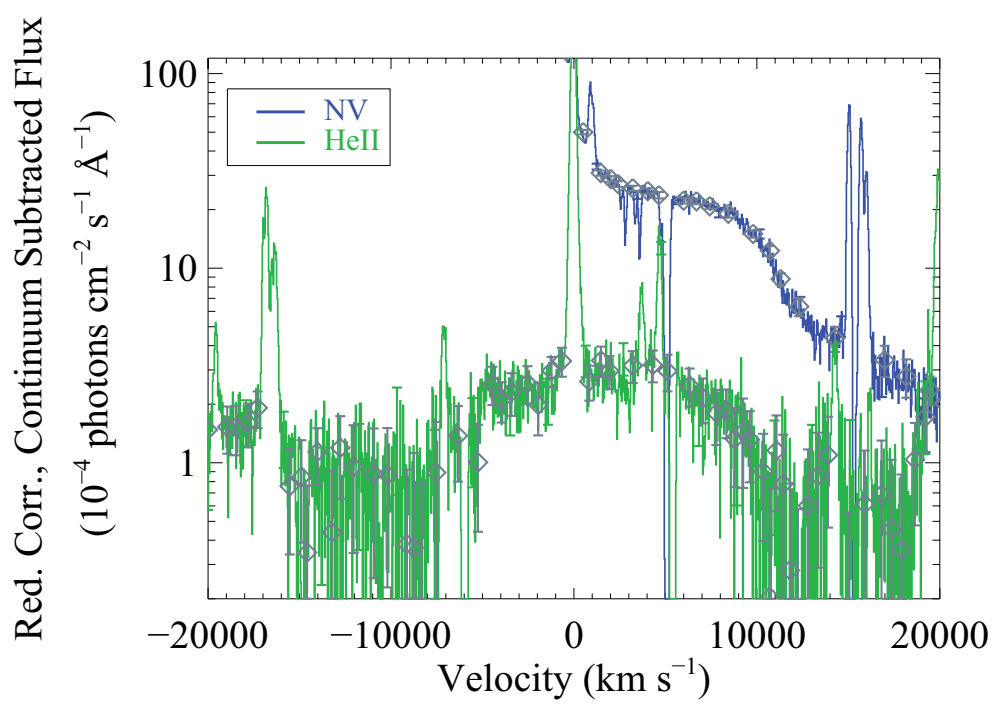

Figure 3. 2-photon continuum-subtracted NV and HeII in the extracted, one-dimensional spectra. The blue side of the NV distribution is lost under the RS Ly $\alpha$ emission. The HeII velocity profile appears qualitatively similar to that of NV.

should be dramatically suppressed (France et al. 2011) and therefore we conclude that the 2-photon emission continuum is dominated by the lower-density outer debris.

Is it reasonable to expect this level of X-ray heating of gas near the reverse shock? We calculate the total X-ray flux from the circumstellar ring using the two-component model spectrum of Zhekov et al. (2006), scaled to the total $0.5-2.0 \mathrm{keV}$ luminosity observed by Chandra near day $8000\left(L(0.5-2.0 \mathrm{keV}) \approx 1.5 \times 10^{36} \mathrm{erg} \mathrm{s}^{-1}\right.$; Racusin et al. 2009). The total 2-photon luminosity is $L(2 s) \approx 2.2 \times 10^{36} \mathrm{erg} \mathrm{s}^{-1}$, therefore the $0.5-2.0 \mathrm{keV}$ X-ray flux is insufficient to power the far-UV continuum. However, the total shock luminosity is most likely dominated by emission in the soft X-ray/EUV band $(0.01-0.5 \mathrm{keV})$ that is attenuated by the neutral hydrogen in the interstellar media of the Milky Way and LMC (Fransson et al. 2011). The luminosity in this band inferred from the model by Zhekov et al. is $L(0.01-0.5 \mathrm{keV}) \approx 3 \times 10^{38} \mathrm{erg} \mathrm{s}^{-1}$. Assuming that roughly half of this emission intersects the outer ejecta, we find that approximately $1.4 \%$ of the soft X-ray/EUV luminosity from the shocked ring must be reprocessed into $\mathrm{H} \mathrm{I}$ 2-photon emission.

\section{High-velocity Metal Emission Lines and CNO Processing in the Progenitor}

Figure 2 shows the broad emission lines of N V, N IV], C IV, and He II imposed on the 2-photon continuum. Prior to the first STIS observations of SN 1987A, Borkowski et al. (1997) predicted that strong reverse shock emission from Li-like N V $\lambda 1240$ would be detectable. This emission was not apparent, however, in the first deep far-UV STIS spectra presented by Sonneborn et al. (1998) and Michael et al. (1998). In recent (January 2010) STIS observations, we tentatively detected broad, redshifted N V emission (Figure 4), but low S/N precluded a detailed analysis. Now, in our COS observations, we unambiguously detect this emission. While the blue wing of the line is lost under the bright Ly $\alpha$ emission, we observe the complete red wing of the $\mathrm{N} \mathrm{V} \lambda 1240$ velocity profile. In Figure 3, we compare the line profile of $\mathrm{N} \mathrm{V}$ with that of He II, observing that the red 

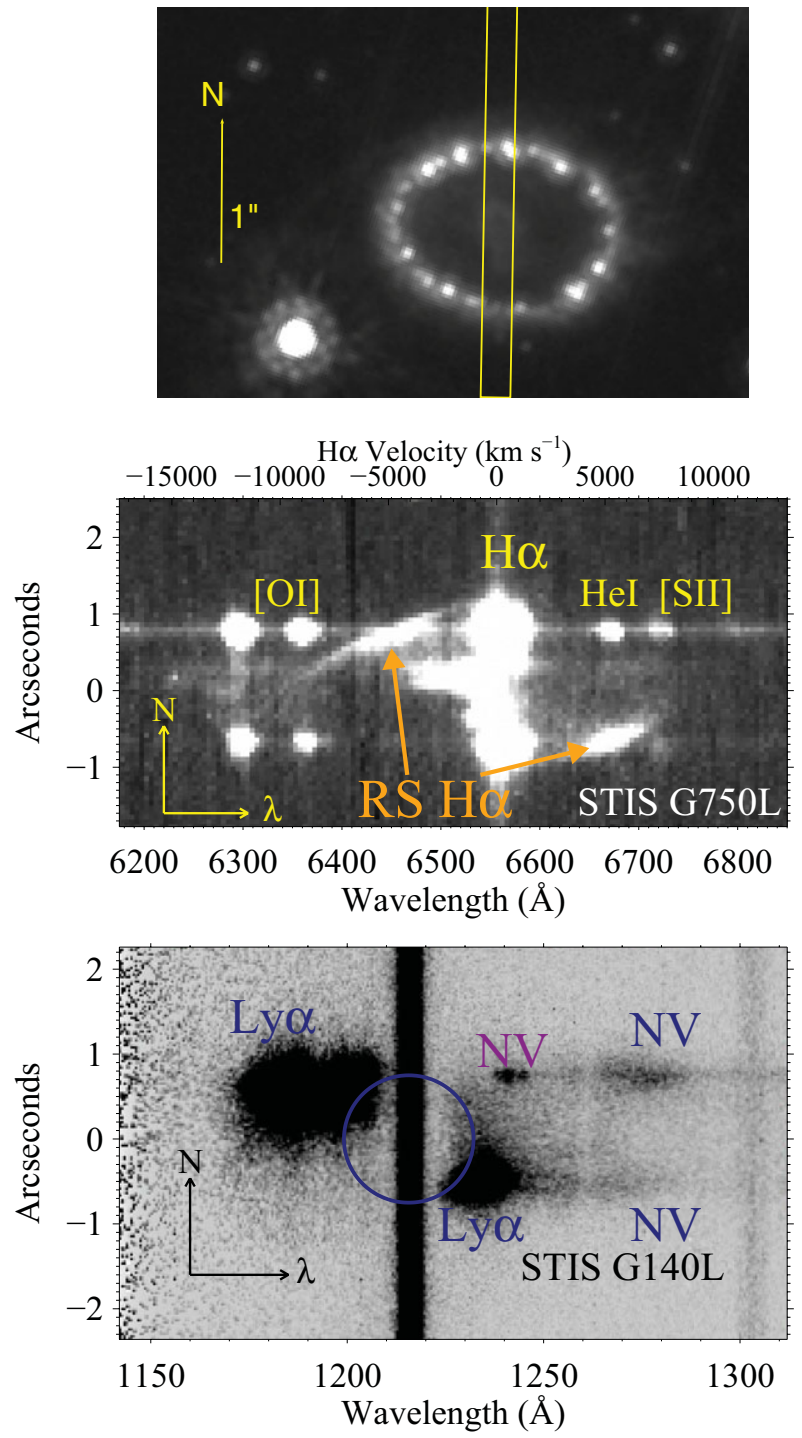

Figure 4. Long-slit imaging spectroscopy from the January 2010 HST observing campaign. top) HST-Advanced Camera for Surveys image obtained on 28 November 2003 in the F625W filter with an exposure time of 800s, illustrating the slit orientation used. (middle) STIS STIS G750L spectrum of SN 1987A, centered about the $\mathrm{H} \alpha$ emission line. The vertical bar at the center of the image is stationary $\mathrm{H} \alpha$ emission from interstellar or circumstellar gas. The bright spots at the north and south of this bar are the emissions from $\mathrm{H} \alpha+[\mathrm{N} \mathrm{II}] \lambda \lambda 6548,6583 \AA$ from hotspots on the equatorial ring. [O I] $\lambda \lambda 6300,6364$, He I $\lambda 6678$, and [S II] $\lambda \lambda 6716,6731$ $\AA$ emission from the hotspots is also observed. The blueshifted streaks near the center are $\mathrm{H} \alpha$ emission excited by radioactivity in the interior of the supernova debris. The curved, blueshifted streak extending from the north side of the vertical bar and the redshifted streak on the south side (noted with orange arrows) are $\mathrm{H} \alpha$ emission from the brightest parts of the reverse shock. bottom STIS G140L observations of SN 1987A. The bright vertical stripe is the slit image in geocoronal Ly $\alpha$. The blue ellipse approximates the location of the circumstellar emission ring. Broad, faint features seen on the north and south sides between $\sim 1260-1290 \AA$ (labeled N V in blue) are produced by nitrogen ions in the reverse shock, and the shock-excited $\mathrm{N} v$ hotspot emission is labeled in magenta. 
wings of the two profiles are qualitatively similar. Both ions present a boxy line profile, with a flat top and fall off between $+9000-+10000 \mathrm{~km} \mathrm{~s}^{-1}$. The $\mathrm{N} \mathrm{V}$ profile extends to at least $+14000 \mathrm{~km} \mathrm{~s}^{-1}$, where the data are contaminated by the geocoronal O I triplet.

We also detect $\mathrm{C}$ IV $\lambda 1550$ from the reverse shock covering $\sim 1500-1580 \AA$, with emission from $-12000--1500 \mathrm{~km} \mathrm{~s}^{-1}$ having 2.2 times the total integrated photon flux as the $+1500-+8000 \mathrm{~km} \mathrm{~s}^{-1}$ interval (Table 2 ). The red side of the velocity profile is only marginally above the noise level at $v_{C I V}>+3000 \mathrm{~km} \mathrm{~s}^{-1}$.

Interpolating the spectrum through the narrow emission lines, we measure an integrated broad line photon flux $\left(-12000-+8000 \mathrm{~km} \mathrm{~s}^{-1}\right)$ of $F(\mathrm{C} \mathrm{IV})=7.7 \times 10^{-3}$ photons $\mathrm{cm}^{-2} \mathrm{~s}^{-1}$. With the previously-noted assumptions about the $\mathrm{N} \mathrm{V}$ flux in mind, we find a flux ratio of $F(\mathrm{~N} V) / F(\mathrm{C} I V) \approx 20 \pm 3$. The ratio of $\mathrm{N} V \lambda 1240 / \mathrm{C}$ IV $\lambda 1550$ is given by an expression similar to equation (2):

$$
\frac{F(N V)}{F(C I V)}=\frac{x_{N}}{x_{C}}\left[\frac{R(1240)}{R\left(N^{4+} \rightarrow N^{5+}\right)}\right]\left[\frac{R(1550)}{R\left(C^{3+} \rightarrow C^{4+}\right)}\right]^{-1}
$$

The ratio of the quantities in square brackets is very close to 0.9 for $v_{s}=(5-12) \times 10^{3}$ $\mathrm{km} \mathrm{s}^{-1}$ (the ratio is near unity because both are Li-like ions). That implies that the abundance ratio of nitrogen/carbon atoms crossing the reverse shock is $x_{N} / x_{C} \approx 22 \pm 3$, greater than the ratio $x_{N} / x_{C} \approx 8.5 \pm 3.5$ inferred from spectroscopic observations of the equatorial ring (Lundqvist \& Fransson 1996; Mattila et al. 2010).

We note that while our estimate of the flux ratio N V $\lambda 1240 / C$ IV $\lambda 1550$ is somewhat uncertain because our choice of the blue side of the $\mathrm{N} \mathrm{V}$ profile is speculative, the integrated flux in the red side of the $\mathrm{N} \mathrm{V}$ profile alone is more than 10 times greater than the total C IV flux. Therefore, we are confident that the N V/CIV ratio is enhanced well beyond what can be attributed to the circumstellar ring abundances of the two species.

\section{Future Work}

One unresolved issue from the studies described above is the large $\mathrm{N} \mathrm{V/C} \mathrm{IV} \mathrm{ratio} \mathrm{in}$ the reverse shock emission. Carbon and nitrogen abundance ratios in the circumstellar ring suggest this number should be $\approx 8$ (Lundqvist \& Fransson 1996; Mattila et al. 2010), however the observed flux ratios indicate $\mathrm{N} / \mathrm{C} \approx 22$. It may be that the $\mathrm{N} / \mathrm{C}$ abundance ratio in the outer envelope of the progenitor was stratified prior to the ejection of the circumstellar rings, and that we are now seeing the first observational evidence of that stratification. A second possibility to account for this discrepancy is that ongoing thermonuclear processing continued to convert $\mathrm{C}$ to $\mathrm{N}$ in the supernova progenitor following the circumstellar ring ejection. The CNO bi-cycle will enrich the ${ }^{14} \mathrm{~N}$ abundance at the expense of the abundances of ${ }^{12} \mathrm{C}$ and ${ }^{16} \mathrm{O}$ and, in equilibrium, will convert almost all of the primordial C and O into N (Caughlan \& Fowler 1962). CNO processing has been invoked to explain the fact that the observed nitrogen abundance in the circumstellar ring is elevated by a factor $\sim 10$ over its value in the LMC (Fransson et al. 1989). Our observation that the He abundance does not change between the ring and reverse shock is qualitatively consistent with the 14E1 model presented by Shigeyama \& Nomoto (1990). They show that the high-velocity material we observe crossing the reverse shock front is only a small fraction of the total ejected mass, and does not probe deep enough into the interior of the ejecta to observe significantly elevated He abundances.

If CNO processing continued near the stellar surface following the ejection of the circumstellar rings, it could have in principle converted most of the remaining $\mathrm{C}$ and $\mathrm{O}$ abundances seen in the equatorial ring into N. Heng et al. (2008) note reduced oxygen 
abundances, possibly related to the high N V/C IV ratio observed in our observations. For this explanation to be viable, the timescale to reach equilibrium in the CNO cycle must be $\lesssim 20,000$ years, the interval since the ejection of the equatorial ring. Additional modeling of the progenitor, ejecta, and reverse shock would be useful for better understanding the large N V/C IV ratio that has been observed in our deep ultraviolet spectra of SN 1987A.

\section{Acknowledgements}

I would like to thank Dick McCray for the opportunity to study SN 1987A; Dick's enthusiasm and vast knowledge of supernova physics have made this collaboration a highlight of my research program. I also acknowledge enjoyable collaboration with the SAINTS team, in particular Bob Kirshner, Pete Challis, and Claes Fransson, during the course of this work. I appreciate the hard work invested by Steve Penton to produce the custom extractions from these challenging COS observations. The SAINTS team thanks Svetozar Zhekov for making his X-ray shock model available, and I also thank James Green for enjoyable discussions about the spectroscopic imaging capabilities of COS.

\section{References}

Callaway, J. 1988, PRA, 37, 3692

Caughlan, G. R. \& Fowler, W. A. 1962, ApJ, 136, 453

Danforth, C. W., Keeney, B. A., Stocke, J. T., Shull, J. M., \& Yao, Y. 2010, ApJ, 720, 976

France, K., Beasley, M., Keeney, B. A., Danforth, C. W., Froning, C. S., Green, J. C., \& Shull, J. M. 2009, ApJl, 707, L27

France, K., McCray, R., Heng, K., Kirshner, R. P., Challis, P., Bouchet, P., Crotts, A., Dwek, E., Fransson, C., Garnavich, P. M., Larsson, J., Lawrence, S. S., Lundqvist, P., Panagia, N., Pun, C. S. J., Smith, N., Sollerman, J., Sonneborn, G., Stocke, J. T., Wang, L., \& Wheeler, J. C. 2010, Science, 329, 1624

France, K., McCray, R., Penton, S. V., Kirshner, R. P., Challis, P., Laming, J. M., Bouchet, P., Chevalier, R., Garnavich, P. M., Fransson, C., Heng, K., Larsson, J., Lawrence, S., Lundqvist, P., Panagia, N., Pun, C. S. J., Smith, N., Sollerman, J., Sonneborn, G., Sugerman, B., \& Wheeler, J. C. 2011, ApJ, 743, 186

Fransson, C., Cassatella, A., Gilmozzi, R., Kirshner, R. P., Panagia, N., Sonneborn, G., \& Wamsteker, W. 1989, ApJ, 336, 429

Fransson, C., Larsson, J., Spyromilio, J., Chevalier, R., Groningsson, P., Jerkstrand, A., Leibundgut, B., McCray, R., Challis, P., Kirshner, R. P., Kjaer, K., Lundqvist, P., \& Sollerman, J. 2012, ArXiv e-prints

Heng, K., Haberl, F., Aschenbach, B., \& Hasinger, G. 2008, ApJ, 676, 361

Heng, K. \& McCray, R. 2007, ApJ, 654, 923

Heng, K., McCray, R., Zhekov, S. A., Challis, P. M., Chevalier, R. A., Crotts, A. P. S., Fransson, C., Garnavich, P., Kirshner, R. P., Lawrence, S. S., Lundqvist, P., Panagia, N., Pun, C. S. J., Smith, N., Sollerman, J., \& Wang, L. 2006, ApJ, 644, 959

Kozma, C. \& Fransson, C. 1992, ApJ, 390, 602

Lundqvist, P. \& Fransson, C. 1996, ApJ, 464, 924

Mattila, S., Lundqvist, P., Gröningsson, P., Meikle, P., Stathakis, R., Fransson, C., \& Cannon, R. 2010, ApJ, 717, 1140

Michael, E., McCray, R., Borkowski, K. J., Pun, C. S. J., \& Sonneborn, G. 1998, ApJ, 492, L143

Michael, E., McCray, R., Chevalier, R., Filippenko, A. V., Lundqvist, P., Challis, P., Sugerman, B., Lawrence, S., Pun, C. S. J., Garnavich, P., Kirshner, R., Crotts, A., Fransson, C., Li, W., Panagia, N., Phillips, M., Schmidt, B., Sonneborn, G., Suntzeff, N., Wang, L., \& Wheeler, J. C. 2003, ApJ, 593, 809

Osterman, S., Green, J., Froning, C., Béland, S., Burgh, E., France, K., Penton, S., Delker, T., Ebbets, D., Sahnow, D., Bacinski, J., Kimble, R., Andrews, J., Wilkinson, E., McPhate, J., Siegmund, O., Ake, T., Aloisi, A., Biagetti, C., Diaz, R., Dixon, W., Friedman, S., 
Ghavamian, P., Goudfrooij, P., Hartig, G., Keyes, C., Lennon, D., Massa, D., Niemi, S., Oliveira, C., Osten, R., Proffitt, C., Smith, T., \& Soderblom, D. 2011, ApS \& S, 157

Racusin, J. L., Park, S., Zhekov, S., Burrows, D. N., Garmire, G. P., \& McCray, R. 2009, ApJ, 703,1752

Shigeyama, T. \& Nomoto, K. 1990, ApJ, 360, 242

Sonneborn, G., Pun, C. S. J., Kimble, R. A., Gull, T. R., Lundqvist, P., McCray, R., Plait, P., Boggess, A., Bowers, C. W., Danks, A. C., Grady, J., Heap, S. R., Kraemer, S., Lindler, D., Loiacono, J., Maran, S. P., Moos, H. W., \& Woodgate, B. E. 1998, ApJl, 492, L139+ Spitzer, L. 1978, Physical processes in the interstellar medium

Xu, Y., McCray, R., Oliva, E., \& Randich, S. 1992, ApJ, 386, 181

Zhekov, S. A., McCray, R., Borkowski, K. J., Burrows, D. N., \& Park, S. 2006, ApJ, 645, 293

\section{Discussion}

PodsiadLOWSKI: You argued that the high N/C ratio in the reverse shock indicates that there must have been significant CNO processing after the inner ring ejection. This of course strongly constrains evolutionary models. I have not looked at this specifically in my own models, but I know that the temperature in the H-burning shell in a post-merger model is high. So the conditions will be right somewhere, but to make this quantitative one needs the total amount that has been processed and also how this is mixed into the region where it is observed (as the star will have a radiative envelope in that phase). What are you going to do about this?

FRANCE: The authors have discussed this problem with Alex Heger, and have discussed the possibility of making a follow-up study comparing stellar models and HST observations. Admittedly, this has not gone anywhere in over a year. I would be happy to work with stellar modelers who have definitive predictions for $\mathrm{N}$ and $\mathrm{C}$ abundances so that we could compare them to observations.

SANKRIT: Can you detect any spatial variation of $\mathrm{NV} / \mathrm{CIV}$, or $\mathrm{YN} / \mathrm{XC}$, (or XN/YN) around the Ring/Ejecta?

FRANCE: With the limited angular resolution of HST-COS $\left(\sim 0.8^{\prime \prime}-1.0^{\prime \prime}\right)$, we can separate the blue vs. red sides of the reverse shock emission; albeit with some overlap. We do see quantitatively and qualitatively different profiles on the red and blue sides in the different lines. However, the broad Reverse Shock Lines (except for Ly $\alpha$ and the red NII) are quite faint. Attempts to look at variations when breaking up the data into subregions results in plots too noisy to be useful.

Chevalien: Where do we stand with regard to determining the geometry of the RS, particularly out of the ring plane.

FRANCE: At present, we have slices of the RS. To understand the true, 3D geometry of the RS, one would want to step the $52 \times 0.2^{\prime \prime}$ STIS slit (6140L) across the circumstellar ring. The problem is that the ring is $\sim 2^{\prime \prime}$ in diameter. So that is 10 STIS pointings X $3-4$ orbits per pointing, and you have 30-40 orbits. A political challenge for an HST Panel. However, we do have good evidence that the RS extends beyond / out of the ring plane. At the time of the HST-COS observations, the expected RS velocity was $\sim 7000 \mathrm{~km} / \mathrm{s}$, however we observed Ly $\alpha$ (blue) and NII (red) in excess of $12,000 \mathrm{~km} / \mathrm{s}$. Since $V \propto r / t$, this suggests that part of the emission comes from $r_{R S}>r_{C S}$, or out of the ring plane. 